\title{
Cocriação de Consumidores do Brasil e Estados Unidos a partir da Análise do Comportamento de duas Sociedades com Traços Culturais de Alto e Baixo Contexto
}

\author{
Co-Creation of Consumers from Brazil and the United States based on the Analysis of the \\ Behavior of two Societies with Cultural Traits of High and Low Context
}

\author{
Nicole Speguem Muratore ${ }^{1}$ \\ Fernanda Lazzari² \\ Juliana Matte ${ }^{3}$ \\ Vandoir Welchen ${ }^{4}$
}

\begin{abstract}
Resumo
O objetivo deste artigo foi analisar o comportamento dos consumidores de dois países (Brasil e EUA) com traços culturais distintos e inseridos em um cenário de cocriação. Para isso, foi realizada uma pesquisa quantitativa, por meio de questões de perfil e de consumo, engajamento, antecipação da satisfação e intenção de compra, no contexto de customização de um tênis. Foram escolhidos EUA e Brasil como países com perfis culturais de baixo contexto e alto contexto, respectivamente. Foram obtidos 405 casos válidos distribuídos entre os dois países (147 brasileiros e 258 norte-americanos), separados em quatro subgrupos diferentes: brasileiros com experiência prévia de cocriação (34), sem experiência prévia de cocriação (113), norte-americanos com experiência prévia de cocriação (71) e sem experiência prévia de cocriação (187). Os resultados apontaram que brasileiros são significantemente mais engajados em comparação aos norte-americanos. Contudo, os norte-americanos apresentaram médias mais altas com relação à antecipação da satisfação e a intenção de compra. Além disso, observou-se que a experiência prévia de cocriação do consumidor não conduziu a respostas comportamentais distintas.
\end{abstract}

Palavras-chave: Cocriação; Engajamento; Antecipação da satisfação; Intenção de compra; Comportamento do consumidor.

\begin{abstract}
Abtract
This paper aimed to analyze the behavior of consumers from two countries (Brazil and the USA) with distinct cultural traits inserted in a co-creation scenario. For this, quantitative research was conducted through questions about profile and consumption, engagement, the anticipation of satisfaction, and purchase intention, in the context of customization of a shoe. The USA and Brazil were chosen as countries with low context and high context cultural profiles, respectively. We obtained 405 valid cases distributed between the two countries (147 Brazilians and 258 US citizens), separated into four different subgroups: Brazilians with prior cocreation experience (34), without prior co-creation experience (113), US citizens with experience prior co-creation (71) and no prior co-creation experience (187). The results showed that Brazilians are significantly more engaged compared to US citizens. However, US citizens scored higher on anticipation of satisfaction and purchase intent. In addition, it was observed that previous consumer co-creation experiences did not lead to distinct behavioral responses.
\end{abstract}

Keywords: Co-creation; Engagement; Anticipation of satisfaction; Buy intention; Consumer behavior.

9 Bacharela em Comércio Internacional pela Universidade de Caxias do Sul (UCS) e Pós-graduanda em Varejo Físico e Online pela Universidade de São Paulo (USP-ESALQ). Doutora em Administração pela Universidade Federal do Rio Grande do Sul (UFRS). Professora e pesquisadora do Programa de Pós-Graduação em Administração da Universidade de Caxias do Sul (UCS). Doutora em Administração pela Universidade de Caxias do Sul. Pesquisadora e Cientista de Dados. 


\section{INTRODUÇÃO}

A imagem de um mercado no qual o contato direto entre empresa e consumidor acontece apenas no momento da compra está mudando frente ao surgimento de consumidores exponencialmente mais conectados (à internet) e informados (PRAHALAD; RAMASWAMY, 2004). Ainda, segundo os autores, o próprio mercado está se tornando um fórum para conversas e interações entre as principais partes envolvidas no negócio: consumidores e comunidades de consumidores, e empresas. Destaca-se o papel promissor que os consumidores podem vir a desempenhar num mundo previamente dominado pela empresa de desenvolvimento e produção (FÜLLER, 2010), evoluindo de um estado de atuação passivo para um ativo (JIMÉNEZ-BARRETO; CAMPO-MARTíNEZ, 2018).

Essa atuação ativa do consumidor está relacionada ao fato de que a simples troca de bens tangíveis já não é suficiente para descrever a relação entre empresas e consumidores, que é cada vez mais centrada na interação e diálogo entre as partes (PAYNE et al., 2009), bem como na capacidade dos clientes de interpretarem os resultados das suas próprias experiências de consumo (JAAKKOLA; HELKKULA; AARIKKA-STENROOS, 2015). O conceito da lógica dominante de serviço, no qual conhecimentos e recursos são compartilhados e a geração de valor é mútua (MATHIS et al., 2016), afirma, em uma de suas principais premissas, que "o consumidor é sempre um cocriador de valor" (VARGO; LUSCH, 2008).

A importância da dimensão experiencial do comportamento do consumidor (JAAKKOLA; HELKKULA; AARIKKASTENROOS, 2015) é destacada a partir do estudo de Vargo e Lusch (2008), uma vez que o valor é determinado unicamente por meio da percepção do beneficiário no contexto das suas interações e experiências com o produto e com a marca. No que diz respeito ao cenário proposto para este estudo, France et al. (2018) definem o comportamento de cocriação do cliente como um processo associado ao relacionamento recíproco com a empresa, considerado ativo, interativo e voluntário. Quando esse relacionamento é positivo e possibilita uma experiência de cocriação atraente para os consumidores participantes, atitudes como satisfação (LÓPEZ; MAYA; LÓPEZ, 2017) e engajamento com a marca (FINSTERWALDER, 2018) podem ser facilmente percebidas ao longo da atividade.

Apesar da cocriação de valor implicar o envolvimento pessoal do consumidor com as atividades promovidas pela empresa, pode-se considerar, segundo os estudos da teoria da cultura do consumo (ARNOULD; THOMPSON, 2005), a experiência como uma construção coletiva e um fenômeno social (HELKKULA, 2011), uma vez que os indivíduos costumam compartilhar suas experiências de cocriação com outras pessoas (LÓPEZ; MAYA; LÓPEZ, 2017). Assim, esta pesquisa tem como objetivo analisar as respostas comportamentais de consumidores de dois países (Brasil e EUA) com traços culturais distintos inseridos em um cenário de cocriação.

Desse modo, a partir desse objetivo, analisou-se o perfil dos indivíduos com e sem experiência prévia de cocriação nos dois grupos culturais estudados; mensurou-se o engajamento, analisou-se a antecipação da satisfação e analisou-se a intenção de compra dos consumidores num cenário de cocriação; bem como comparou-se a percepção dos dois grupos culturais estudados em relação aos construtos investigados.

\section{REFERENCIAL TEÓRICO}

\subsection{Culturas de alto e de baixo contexto}

As pessoas que integram uma sociedade são formadas por três níveis diferentes (e hierárquicos): natureza humana, cultura e personalidade. A cultura é um fenômeno coletivo capaz de diferenciar os membros de um grupo específico do restante dos indivíduos de outros grupos (HOFSTEDE; HOFSTEDE; MINKOV, 2010). O antropólogo americano Hall (1989) define cultura como uma série de modelos situacionais de diferentes níveis de comportamentos, e afirma que os pensamentos e as atitudes são amplamente modificados pelo padrão cultural onde as pessoas estão inseridas. Como uma forma de interpretar diferentes orientações culturais, Hall (1989) propõe o espectro de uma linha contínua, na qual os extremos são alto e baixo contexto, e no qual os países podem ser classificados para representar a extensão em que o contexto ocorre, apesar de nenhuma cultura existir exclusivamente num determinado ponto da escala.

Culturas de alto contexto são caracterizadas por uma comunicação verbalizada simples, mas que contém significado nas entrelinhas da mensagem, uma vez que os indivíduos dessas sociedades cultivam relações pessoais e íntimas. Assim, consequentemente, a sociedade apresenta uma estrutura hierárquica bem definida na qual os sentimentos coletivos são mais valorizados, enquanto a subjetividade é mantida implícita por um forte autocontrole individual (HALL, 1989; NGUYEN; HEELER; TARAN, 2007; JEONG; CROMPTON, 2018).

Por outro lado, culturas de baixo contexto são exatamente o oposto e costumam se comunicar de maneira que o que é dito é exatamente aquilo que significa. Nesse outro polo do espectro, há culturas que a sociedade é mais individualista e fragmentada, e há, relativamente, pouco envolvimento com as demais pessoas. Como resultado disso, a hierarquia social, e até mesmo a comunidade em geral, exercem menos influência sobre a vida e as decisões dos sujeitos, bem como a comunicação tende a ser mais explícita e impessoal (HALL, 1989; NGUYEN; HEELER; TARAN, 2007; JEONG; CROMPTON, 2018). 
Kim, Pan e Park (1998) afirmam que o potencial dos estudos culturais de Edward T. Hall, sobretudo os conceitos de cultura de alto e baixo contexto, são amplamente discutidos e aplicados no âmbito do marketing internacional, uma vez que constituem uma ferramenta para auxiliar no entendimento das diferenças entre culturas. Desse modo, a relação entre cultura e consumo pode ser avaliada sob o ponto de vista de que os aspectos da vida em sociedade e também as dimensões comportamentais do ser humano são amplamente influenciadas por fatores culturais (SOARES; FARHANGMEHR; SHOHAMB, 2007).

\subsection{Cocriação de valor}

A realidade emergente da economia do século XXI está tornando obsoleto o sistema tradicional de criação de valor, centrado na empresa, ao mesmo tempo em que força o surgimento de um novo modelo industrial, voltado para a cocriação de valor aliada à transformação do papel do consumidor nas relações de consumo e produção de bens e serviços (PRAHALAD; RAMASWAMY, 2004). Apesar da popularização da palavra e da ampliação do seu significado, Ramaswamy e Ozcanb (2018) definem cocriação como uma ferramenta de criação através da interação nas suas mais diversas áreas de aplicação. Conforme o relacionamento entre empresas e consumidores se torna complexo - partindo da simplicidade, entre uma empresa e um consumidor; depois centralizado, entre uma empresa e diversos consumidores; e por fim, diversificado, quando os pontos de contato são múltiplos, entre várias empresas e vários consumidores que interagem entre si - cresce a singularidade de valor (PRAHALAD; RAMASWAMY, 2004).

Por meio da ligação entre fornecedor e cliente, a cocriação de valor deve colaborar para o estabelecimento de relações que incentivem esse tipo de experiência com o objetivo de alcançar reciprocamente resultados positivos (BUSSER; SHULGA, 2018), e contribuir para o alcance de uma vantagem competitiva possibilitada pelo favorecimento da participação constante do consumidor nos processos empresariais (COSSíO-SILVA et al., 2016). Complementando, Busser e Shulga (2018) afirmam que, através de contribuições voluntárias de múltiplos atores, a cocriação pode ser entendida como um processo conjunto de produção de novo valor, tanto material quanto simbólico, refletindo perspectivas sociais, colaborativas e compartilhadas nos campos da prestação de serviços ou da fabricação de produtos.

A cocriação de experiências de serviço ocorre, sobretudo, na interação entre o cliente e o próprio prestador de serviços, ou entre o cliente e outros clientes, podendo abranger experiências vividas ou imaginárias no passado, presente e futuro. Tem-se uma experiência de cocriação de serviço a partir do momento que a interação interpessoal com outros atores influencia as respostas subjetivas de um indivíduo ou a interpretação dos elementos do serviço (JAAKKOLA; HELKKULA; AARIKKA-STENROOS, 2015). Análogo a isso, encontra-se a cocriação de produto, que na visão de Ind e Coates (2013) está altamente relacionada à percepção de valor que os consumidores têm da utilidade do produto e dos serviços prestados por ele. Com base na teoria da lógica dominante de serviço, a conexão entre o que a organização oferece ao cliente no momento da compra e o uso pelos consumidores ao longo do tempo (VARGO; LUSCH, 2008).

Segundo Payne, Storbackab e Frow (2008), o processo de cocriação de valor, em setores da economia como serviços e indústria, envolve a criação de valor superior pelo fornecedor, aliado às determinações dos clientes sobre o valor no momento em que um bem ou serviço é consumido. Na visão de Blasco-Arcas, Hernandez-Ortega e JimenezMartinez (2014), essa relação entre cocriação de serviço e cocriação de produto pode ser melhor interpretada no contexto de plataformas de compras on-line, que são favoráveis tanto para promover a relação entre os diferentes atores (caracterizando um serviço), quanto para gerar experiência (de compra de um produto). Assim, para os autores, é possível dividir o processo de cocriação que engloba serviço e produto em experiência de compra e experiência de consumo, respectivamente.

\subsection{Relação entre comportamento do consumidor e cocriação}

Os estudos de Prahalad e Ramaswamy (2004) e Vargo e Lusch (2008) destacam o papel ativo do consumidor na criação de valor, já que esse deixou de ser apenas um objeto das ações de marketing para se tornar um importante colaborador do processo como um todo (COSSíO-SILVA et al., 2016). Anteriormente, a partir de uma lógica dominante de bens, os consumidores eram limitados a adquirir os produtos finais de empresas que detinham os recursos de desenvolvimento e produção (PAYNE et al., 2009). Em contrapartida, propõe-se um comportamento do consumidor orientado pela natureza interativa e de relacionamento da cocriação, voltada para a valorização do consumo e não somente da troca no momento da compra (BLASCO-ARCAS; HERNANDEZ-ORTEGA; JIMENEZ-MARTINEZ, 2014).

Para Yi e Gong (2013), o comportamento do consumidor num cenário de cocriação de valor pode ser analisado partindo de duas dimensões distintas: (i) comportamento de participação do cliente, relacionado às práticas necessárias para o sucesso da prática; e (ii) comportamento de cidadania do cliente, caracterizado como voluntário e extraordinário para o processo. Sob a perspectiva do cliente, os consumidores são motivados a cocriar pelos diferentes benefícios que esperam em troca da sua participação (VERLEYE, 2015). Ademais, a qualidade percebida das ferramentas facilitadoras de cocriação - eletrônicas e virtuais - ofertadas pelas empresas, tem influência na disposição dos usuários de participarem de experiências de cocriação (JIMÉNEZ-BARRETO; CAMPO-MARTÍNEZ, 2018). 
Por fim, Luo, Zhang e Liu (2015) descrevem sobre como as práticas de cocriação de valor, através de troca e interação com os consumidores, são capazes de influenciar as relações e têm ligação direta com as respostas comportamentais e, principalmente, de consumo, dos usuários de determinado produto ou serviço. A seguir são apresentados os construtos de engajamento, antecipação de satisfação e intenção de compra, que foram utilizados para a compreensão da relação entre comportamento do consumidor e cocriação.

\subsubsection{Engajamento}

Considerado como uma prática predominante em diversas áreas, o conceito de engajamento pode e deve ser interpretado como receptivo e transmissivo, estando presente nos campos psicológicos e comportamentais dos indivíduos (FINSTERWALDER, 2018). Sob o âmbito do engajamento do consumidor, Brodie et al. (2011) o definem como sendo um estado de dimensão comportamental que ocorre em virtude de experiências interativas e cocriativas focadas em um objeto, como uma marca ou um produto em relações de serviço.

Alinhado ao discurso de cocriação de valor de Vargo e Lusch (2008), no qual os diferentes tipos de indivíduos são considerados como atores no processo, o conceito de engajamento proposto por Finsterwalder (2018) é inclusivo e abrangente, dissolvendo a divisão do engajamento simplista entre empresa e cliente e focando na interação entre os atores (e recursos) envolvidos. Segundo López, Maya e López (2017), engajar o consumidor em atividades de cocriação reforça o comportamento positivo pré e pós compra, se for considerar a estreita relação entre cocriação de valor e participação ativa. Em outras palavras, os consumidores se mostram satisfeitos com o desempenho de sua cocriação, complementando o valor subjetivo atribuído ao produto. Contudo, é importante salientar que a natureza dinâmica do construto implica na variação do nível de engajamento de um ator ao longo do processo, o que pode exigir um estímulo adicional para iniciar ou aumentar o engajamento do consumidor no início, durante e no final do processo interativo (FINSTERWALDER, 2018).

\subsubsection{Antecipação da satisfação}

Segundo Philippe e Guintcheva (2014), a satisfação dos consumidores é pertinente aos aspectos emocionais das expectativas dos mesmos em relação a uma experiência antecipada. No que diz respeito à antecipação da satisfação, os estudos de Shiv e Huber (2000) afirmam que esse tipo de conduta surge comumente em contextos que requerem maior envolvimento do consumidor, ligados à experiência de compras sensoriais. De acordo com Verleye (2015), quanto mais altos forem os níveis de conectividade, mais a antecipação da satisfação dos clientes com os produtos ou serviços recém-gerados é afetada positivamente.

O conceito de cocriação, para além do seu âmbito prático de interação entre diversas partes na cadeia produtiva de valor, também implica no compartilhamento de informações e de experiências dos indivíduos com o restante da sociedade. Para López, Maya e López (2017), o compartilhamento social de experiências de cocriação pode impulsionar diretamente o efeito positivo da cocriação de valor na satisfação do consumidor e, consequentemente, aumentar a intenção de compra do mesmo.

\subsubsection{Intenção de compra}

O comportamento de consumo conhecido como intenção de compra é determinado pela probabilidade e intenção que um indivíduo tem de comprar determinada marca ou produto (BELCH; BELCH, 2004; LU; FAN; ZHOU, 2016), variando de acordo com a experiência, positiva ou negativa, de cada consumidor. Aliada a essa definição, Payne, Storbackab e Frow (2008) sugerem que a participação no processo de criação de valor pode influenciar as decisões de intenção de compra dos consumidores.

Prahalad e Ramaswamy (2004) apontam que um ambiente que favorece a personalização de produtos por parte dos clientes, a realização de interações significativas e a cocriação da experiência de compra, promove atitudes e comportamentos positivos em relação à empresa. Segundo os estudos de Blasco-Arcas, Hernandez-Ortega e JimenezMartinez (2014), envolver os clientes no design do produto ou serviço antes e durante a compra, possibilitando-os adaptar a oferta de acordo com as suas próprias preferências, motiva a participação de consumidores em processos cocriativos e incentiva as intenções de compra dos mesmos.

No campo da cocriação de valor, a experiência de compra não deve ser considerada apenas como um estágio transacional que envolve somente a troca de um produto e/ou dinheiro entre os participantes, mas também deve ser valorizada como uma atividade social que contribui para o desenvolvimento e manutenção de relacionamentos significativos entre os clientes e a empresa (BLASCO-ARCAS; HERNANDEZ-ORTEGA; JIMENEZ-MARTINEZ, 2014). 


\section{PROCEDIMENTOS METODOLÓGICOS}

A pesquisa possui uma abordagem quantitativa de caráter descritivo, com base em uma coleta de dados estruturada (SAMARA; BARROS, 2007), do tipo survey (KLEIN et al., 2015). A escolha do público alvo da pesquisa se baseou no estudo sobre diferenças culturais de Hall (1989), que propõe a classificação dos países em culturas de alto e baixo contexto, desse modo, portanto, optou-se por aplicar a pesquisa nos EUA e no Brasil.

A primeira parte do questionário possuiu questões de caracterização da amostra, sendo, subsequentemente, apresentadas perguntas para traçar o perfil de consumo do indivíduo, ou seja, questões-filtro para checar se os respondentes compreenderam o significado de cocriação e se os mesmos já tiveram alguma experiência efetiva de cocriação. Ainda, para evitar possíveis desvios na pesquisa, um texto descrevendo uma situação hipotética foi elaborado, dentro do contexto de customização de um tênis, a fim de direcionar o pensamento dos respondentes acerca do mesmo produto/serviço em análise. Desse modo, o cenário descrito foi baseado no estudo de BlascoArcas, Hernandez-Ortega e Jimenez-Martinez (2014).

Posteriormente, aplicaram-se as escalas de engajamento de Cian, Krishna e Elder (2014), de antecipação da satisfação, adapatada de Shiv e Huber (2000) e de intenção de compra de Bone e Ellen (1992), todas medidas a partir da escala do tipo Likert de 5 pontos, que variava entre 1 (discordo totalmente) e 5 (concordo totalmente). Em virtude da aplicação da pesquisa em dois países diferentes, os respondentes receberam o mesmo questionário apenas com variação de idioma: português (Brasil) e inglês (EUA). Uma vez que as escalas que compõem o instrumento são originalmente escritas na língua inglesa, as questões foram submetidas à técnica de tradução reversa para o português, evitando, assim, incoerências e quaisquer divergências de interpretação.

A coleta de dados ocorreu eletronicamente, por meio da plataforma Google Forms, nas versões dos dois idiomas dos grupos foco de análise. No Brasil, o link do questionário em português foi enviado para aplicativos de mensagens, redes sociais e e-mail. Nos EUA, a ferramenta de coleta foi a Mechanical Turk. A coleta dos dados ocorreu no mês de outubro de 2018 e foram obtidas 585 respostas, sendo 227 do Brasil (Grupo 1) e 368 dos EUA (Grupo 2).

Após a coleta, os dados foram tabulados em planilha do Microsoft Excel ${ }^{\circledR}$, onde foi possível identificar respostas atípicas e possíveis outliers, resultando no total de 405 casos válidos, 147 do Grupo 1 (G1) e 258 do Grupo 2 (G2). A análise de dados ocorreu por meio da estatística descritiva, teste $t$ - técnica de estatística paramétrica -, para testar diferenças significantes de médias entre os grupos pesquisados e comparar as duas amostras independentes (HAIR et al., 2005), com o auxílio do software de análise estatística SPSS ${ }^{\circledR} 21$.

\section{APRESENTAÇÃO E ANÁLISE DOS RESULTADOS}

\subsection{Perfil dos respondentes}

A partir da análise das perguntas sobre perfil demográfico e de consumo do questionário, foi possível identificar as características das amostras dos Grupos G1 e G2. Em um primeiro momento, os dois grupos das nacionalidades foram subdivididos em quatro grupos menores, baseando-se no fato de o respondente já ter tido uma experiência prévia de cocriação ou não. Assim, o total de casos válidos para o Grupo 1 foi separado em: Grupo 1.1, para brasileiros que já experimentaram a cocriação (34); e Grupo 1.2, para brasileiros que nunca experimentaram a cocriação (113). Ao mesmo tempo, o Grupo 2 foi segregado em Grupo 2.1, norte-americanos que já experienciaram a cocriação (71) e Grupo 2.2, definindo os norte-americanos que nunca tiveram uma experiência de cocriação (187).

A média de idade total, somando os 405 respondentes é de 29,65 anos, sendo que desses o $\mathrm{G} 1$ apresenta média de 22,84 e o G2 média de 33,53. Ao aprofundar a análise do Grupo 1 (brasileiros), verificou-se que a média de idade do G1.1 é de 24,09 anos, enquanto a média do G1.2 é de 22,47 anos. No caso do Grupo 2 (norte-americanos), constatou-se que a média de idade do G2.1 é de 31,83 e a média do G2.2 é de 34,17 anos. Assim, a Tabela 1 apresenta as características das amostras obtidas no que diz respeito ao perfil demográfico dos indivíduos brasileiros e norte-americanos. 
Tabela 1 - Perfil demográfico dos respondentes

\begin{tabular}{|c|c|c|c|c|c|c|}
\hline \multirow{2}{*}{ QUESTÕES } & \multicolumn{3}{|c|}{ BRASIL } & \multicolumn{3}{|c|}{ EUA } \\
\hline & G1 & G1.1 & G1.2 & G2 & G2.1 & G2.2 \\
\hline \multicolumn{7}{|l|}{ GÊNERO } \\
\hline Masculino & $55(37,4 \%)$ & $17(50 \%)$ & $38(33,6 \%)$ & $114(44,2 \%)$ & $31(43,7 \%)$ & $83(44,4 \%)$ \\
\hline Feminino & $92(62,6 \%)$ & $17(50 \%)$ & $75(66,4 \%)$ & $144(55,8 \%)$ & $40(56,3 \%)$ & $104(55,6 \%)$ \\
\hline Total & $147(100 \%)$ & $34(100 \%)$ & $113(100 \%)$ & $258(100 \%)$ & $71(100 \%)$ & $187(100 \%)$ \\
\hline \multicolumn{7}{|l|}{ ESCOLARIDADE } \\
\hline Menos de 10 anos & $1(0,7 \%)$ & $0(0 \%)$ & $1(0,9 \%)$ & $11(4,3 \%)$ & $8(11,3 \%)$ & $3(1,6 \%)$ \\
\hline 11 anos & $5(3,4 \%)$ & $2(5,9 \%)$ & $3(2,7 \%)$ & $5(1,9 \%)$ & $3(4,2 \%)$ & $2(1,1 \%)$ \\
\hline 12 anos & $9(6,1 \%)$ & $1(2,9 \%)$ & $8(7,1 \%)$ & $30(11,6 \%)$ & $10(14,1 \%)$ & $20(10,7 \%)$ \\
\hline 13 anos & $11(7,5 \%)$ & $3(8,8 \%)$ & $8(7,1 \%)$ & $14(5,4 \%)$ & $4(5,6 \%)$ & $10(5,3 \%)$ \\
\hline 14 anos & $15(10,2 \%)$ & $1(2,9 \%)$ & $14(12,4 \%)$ & $38(14,7 \%)$ & $9(12,7 \%)$ & $29(15,5 \%)$ \\
\hline 15 anos & $25(17,0 \%)$ & $5(14,7 \%)$ & $20(17,7 \%)$ & $26(10,1 \%)$ & $6(8,5 \%)$ & $20(10,7 \%)$ \\
\hline 16 anos & $31(21,1 \%)$ & $7(20,6 \%)$ & $24(21,2 \%)$ & $63(24,4 \%)$ & $15(21,1 \%)$ & $48(25,7 \%)$ \\
\hline 17 anos & $22(15,0 \%)$ & $7(20,6 \%)$ & $15(13,3 \%)$ & $28(10,9 \%)$ & $4(5,6 \%)$ & $24(12,8 \%)$ \\
\hline Mais de 18 anos & $28(19,0 \%)$ & $8(23,5 \%)$ & $20(17,7 \%)$ & $43(16,7 \%)$ & $12(16,9 \%)$ & $31(16,6 \%)$ \\
\hline Total & $147(100 \%)$ & $34(100 \%)$ & $113(100 \%)$ & $258(100 \%)$ & $71(100 \%)$ & $187(100 \%)$ \\
\hline \multicolumn{7}{|l|}{ RENDA } \\
\hline Até $\mathrm{R} \$ 708,19 / \$ 230,68$ & $0(0,0 \%)$ & $0(0,0 \%)$ & $0(0,0 \%)$ & $7(2,7 \%)$ & $1(1,4 \%)$ & $6(3,2 \%)$ \\
\hline$R \$ 708,20$ - $R \$ 1.691,44 / \$ 230,69$ - $\$ 550,96$ & $6(4,1 \%)$ & $1(2,9 \%)$ & $5(4,4 \%)$ & $22(8,5 \%)$ & $13(18,3 \%)$ & $9(4,8 \%)$ \\
\hline $\mathrm{R} \$ 1.691,45$ - $\mathrm{R} \$ 2.965,69 / \$ 550,97$ e $\$ 966,02$ & $18(12,2 \%)$ & $5(14,7 \%)$ & $13(11,5 \%)$ & $26(10,1 \%)$ & $11(15,5 \%)$ & $15(8,0 \%)$ \\
\hline$R \$ 2.965,70$ - $R \$ 5.363,19 / \$ 966,03-\$ 1.746,97$ & $46(31,3 \%)$ & $7(20,6 \%)$ & $39(34,5 \%)$ & $42(16,3 \%)$ & $13(18,3 \%)$ & $29(15,5 \%)$ \\
\hline$R \$ 5.363,20$ - $R \$ 10.386,52 / \$ 1,746.98$ - $\$ 3,383.23$ & $53(36,1 \%)$ & $13(38,2 \%)$ & $40(35,4 \%)$ & $59(22,9 \%)$ & $10(14,1 \%)$ & $49(26,2 \%)$ \\
\hline $\mathrm{R} \$ 10.386,53$ - $\mathrm{R} \$ 23.345,11 / \$ 3.383,24-\$ 7.604,27$ & $20(13,6 \%)$ & $6(17,6 \%)$ & $14(12,4 \%)$ & $68(26,4 \%)$ & $15(21,1 \%)$ & $53(28,3 \%)$ \\
\hline Acima de $\mathrm{R} \$ 23.345,12 / \$ 7.604,28$ & $4(2,7 \%)$ & $2(5,9 \%)$ & $2(1,8 \%)$ & $34(13,2 \%)$ & $8(11,3 \%)$ & $26(13,9 \%)$ \\
\hline Total & $147(100 \%)$ & $34(100 \%)$ & $113(100 \%)$ & $258(100 \%)$ & $71(100 \%)$ & $187(100 \%)$ \\
\hline
\end{tabular}

Fonte: elaborado pelos autores (2019)

Com relação ao gênero, o público feminino é predominante nos Grupos G1 (62,6\%) e G1.2 (66,4\%), enquanto que no G1.1 representa exatamente $50 \%$ dos respondentes. Sobre o nível de escolaridade dos respondentes, $21,1 \%$ do G1 e $21,2 \%$ do G1.2 possuem aproximadamente 16 anos de ensino formal; já no caso do G1.1, 23,5\% dos indivíduos possuem mais de 18 anos de ensino formal. Isso significa que, dentre os respondentes brasileiros, aqueles que possuem ensino superior em andamento ou completo estão em maior número. A faixa de renda que ficou em evidência entre os respondentes dos três grupos pesquisados do Brasil foi a quinta faixa entre $\mathrm{R} \$ 5.363,20$ e $\mathrm{R} \$ 10.386,52$.

No caso dos respondentes dos EUA, o público feminino predomina nos três grupos de norte-americanos (55,8\% em G2, 56,3\% em G2.1 e 55,6\% em G2.2). Quanto à escolaridade, assim como no Brasil, o maior índice de respostas sugere que os pesquisados têm 16 anos de ensino formal (G2=24,4\%, G2.1=21,1\% e G2.2=25,7\%), ou seja, possuem ensino superior em andamento ou completo. Tratando-se da renda, $26,4 \%$ do G2, $21,1 \%$ do G2.1 e $28,3 \%$ do G2.2 se encaixam na sexta faixa, entre $\$ 3,383.24$ e $\$ 7,604.27$.

Assim, a realização do teste $t$ para os aspectos de perfil demográfico indicou diferença significante na média de idade entre os Grupos G1 e G2, conforme apresenta a Tabela 2. Por outro lado, as questões acerca de gênero, escolaridade e renda não apontaram diferença significante entre brasileiros e norte-americanos.

Tabela 2 - Teste $t$ das questões de perfil demográfico

\begin{tabular}{lcc}
\hline \multicolumn{1}{c}{ PERFIL } & T & SIGNIFICÂNCIA \\
\hline Gênero & $-1,328$ & 0,185 \\
Idade & $-12,668$ & 0,000 \\
Escolaridade & 1,847 & 0,065 \\
Renda & $-1,953$ & 0,052 \\
\hline
\end{tabular}

Fonte: elaborado pelos autores (2019). 
Dando continuidade à análise dos dados, os respondentes de ambos os grupos e dos quatro subgrupos foram avaliados quanto ao seu perfil de consumo. A fim de caracterizar as amostras, os brasileiros e norte-americanos pesquisados responderam questões em relação à categoria de produto utilizada na situação experimental apresentada no decorrer do questionário. Os resultados das amostras do Brasil (G1, G1.1 e G1.2) e dos EUA (G2, G2.1 e G2.2) estão descritos na Tabela 3, assim como as médias de preço para os três grupos de brasileiros e para os três grupos de norte-americanos.

Tabela 3 - Perfil de consumo dos respondentes brasileiros e norte-americanos

\begin{tabular}{|c|c|c|c|c|c|c|}
\hline \multirow{2}{*}{ QUESTÕES } & \multicolumn{3}{|c|}{ BRASIL } & \multicolumn{3}{|c|}{ EUA } \\
\hline & G1 & G1.1 & G1.2 & G2 & G2.1 & G2.2 \\
\hline \multicolumn{7}{|c|}{ USO SEMANAL DE TÊNIS } \\
\hline Nenhuma vez por semana & $2(1,4 \%)$ & $0(0 \%)$ & $2(1,8 \%)$ & $14(5,4 \%)$ & $2(2,8 \%)$ & $12(6,4 \%)$ \\
\hline 1 vez por semana & $11(7,5 \%)$ & $3(8,8 \%)$ & $8(7,1 \%)$ & $23(8,9 \%)$ & $10(14,1 \%)$ & $13(7,0 \%)$ \\
\hline 2 vezes por semana & $9(6,1 \%)$ & $2(5,9 \%)$ & $7(6,2 \%)$ & $34(13,2 \%)$ & $16(22,5 \%)$ & $18(9,6 \%)$ \\
\hline 3 vezes por semana & $11(7,5 \%)$ & $4(11,8 \%)$ & $7(6,2 \%)$ & $45(17,4 \%)$ & $10(14,1 \%)$ & $35(18,7 \%)$ \\
\hline 4 vezes por semana & $13(8,8 \%)$ & $2(5,9 \%)$ & $11(9,7 \%)$ & $24(9,3 \%)$ & $6(8,5 \%)$ & $18(9,6 \%)$ \\
\hline 5 vezes por semana & $21(14,3 \%)$ & $2(5,9 \%)$ & $19(16,8 \%)$ & $38(14,7 \%)$ & $10(14,1 \%)$ & $28(15,0 \%)$ \\
\hline 6 vezes por semana & $20(13,6 \%)$ & $5(14,7 \%)$ & $15(13,3 \%)$ & $10(3,9 \%)$ & $4(5,6 \%)$ & $6(3,2 \%)$ \\
\hline Todos os dias & $60(40,8 \%)$ & $16(47,8 \%)$ & $44(38,9 \%)$ & $70(27,1 \%)$ & $13(18,3 \%)$ & $57(30,5 \%)$ \\
\hline Total & $147(100 \%)$ & $34(100 \%)$ & $113(100 \%)$ & $258(100 \%)$ & $71(100 \%)$ & $187(100 \%)$ \\
\hline \multicolumn{7}{|c|}{ COMPRA ANUAL DE TÊNIS } \\
\hline Nenhum par & $8(5,4 \%)$ & $2(5,9 \%)$ & $6(5,3 \%)$ & $13(5,0 \%)$ & $2(2,8 \%)$ & $11(5,9 \%)$ \\
\hline 1 par & $63(42,9 \%)$ & $13(38,2 \%)$ & $50(44,2 \%)$ & $83(32,2 \%)$ & $13(18,3 \%)$ & $70(37,4 \%)$ \\
\hline 2 pares & $38(25,9 \%)$ & $9(26,5 \%)$ & $29(25,7 \%)$ & $76(29,5 \%)$ & $21(29,6 \%)$ & $55(29,4 \%)$ \\
\hline 3 pares & $18(12,2 \%)$ & $3(8,8 \%)$ & $15(13,3 \%)$ & $45(17,4 \%)$ & $20(28,2 \%)$ & $25(13,4 \%)$ \\
\hline 4 pares & $13(8,8 \%)$ & $5(14,7 \%)$ & $8(7,1 \%)$ & $21(8,1 \%)$ & $9(12,7 \%)$ & $12(6,4 \%)$ \\
\hline 5 pares & $3(2,0 \%)$ & $0(0 \%)$ & $3(2,7 \%)$ & $14(5,4 \%)$ & $5(7,0 \%)$ & $9(4,8 \%)$ \\
\hline Mais de 5 pares & $4(2,7 \%)$ & $2(5,9 \%)$ & $2(1,8 \%)$ & $6(2,3 \%$ & $1(1,4 \%)$ & $5(2,7 \%)$ \\
\hline Total & $147(100 \%)$ & $34(100 \%)$ & $113(100 \%)$ & $258(100 \%)$ & $71(100 \%)$ & $187(100 \%)$ \\
\hline Preço médio & $\mathrm{R} \$ 238,00$ & $\mathrm{R} \$ 272,00$ & $\mathrm{R} \$ 228,00$ & $\$ 75,00$ & $\$ 86,00$ & $\$ 70,00$ \\
\hline
\end{tabular}

Fonte: elaborado pelos autores (2019).

A respeito da frequência semanal de uso de tênis, 40,8\% dos 147 respondentes brasileiros (G1) afirmaram que calçam tênis todos os dias da semana. Essa porcentagem é ainda maior no grupo daqueles com experiência de cocriação (G1.1), já que dos 34 respondentes, $47,8 \%$, usam tênis todos os dias. Quando questionados sobre a frequência anual de compra de tênis, $42,9 \%$ do G1, 38,2\% do G1.1 e $44,2 \%$ do G1.2 compram apenas um par de tênis por ano. Desses, os que apresentam preço médio mais alto no momento da compra de um par de tênis novo são os representantes do $\mathrm{G} 1.1(\mathrm{R} \$ 272,00)$.

Quando questionados a respeito dos meios pelos quais eles costumam comprar tênis e quais são os atributos que mais valorizam em um modelo do produto em específico, para a maioria dos brasileiros o meio mais comum é comprar tênis em lojas multimarcas, sejam elas físicas ou virtuais $(\mathrm{G} 1=94 \%)$, tendo conforto e preço enquanto as características de produto mais valorizadas $(\mathrm{G} 1=48 \%)$. Já para os norte-americanos, a escolha mais comum é adquirir tênis em lojas multimarcas físicas (58\%), seguida por lojas virtuais exclusivas da marca (39\%). Ainda, 92\% elenca conforto e preço como os atributos principais de um tênis.

Quando questionados a respeito da frequência semanal de uso de tênis, os percentuais mais representativos dentre os respondentes dos Grupos G2 $(27,1 \%)$ e G2.2 (30,5\%) confirmaram que usam tênis todos os dias da semana, apresentando porcentagens superiores quando comparados ao G2.1, onde o maior percentual de indivíduos (22,5\%) utiliza tênis duas vezes por semana. Em relação à frequência de compra anual de tênis por parte dos respondentes norte-americanos, as porcentagens mais altas variam de $32,2 \%$ e $37,4 \%$ nos Grupos G2 e G2.2, respectivamente, indicando que estes compram apenas um par de tênis por ano, para $29,6 \%$ no Grupo G2.1, que compra até dois pares de tênis anualmente. Assim como no caso dos brasileiros, o preço médio mais caro pago por norte-americanos em um par de tênis novo encontra-se no grupo daqueles com experiência prévia de cocriação $(\$ 86,00)$.

Por fim, os grupos pesquisados foram avaliados em relação ao conhecimento prévio do conceito de cocriação. A maioria dos respondentes brasileiros do $\mathrm{G} 1$ (63,3\%), do G1.1 (52,9\%) e do G1.2 (66,4\%) responderam de forma negativa à pergunta, afirmando não conhecerem previamente o conceito após lerem a descrição do mesmo. Ao contrário do $\mathrm{G} 1$, os respondentes do $\mathrm{G} 2$ estão mais familiarizados com o conceito de cocriação, já que $64 \%$ dos 258 norte- 
americanos pesquisados afirmaram conhecê-lo. Esse índice é ainda mais alto no Grupo G2.1, de norte-americanos com experiência prévia de cocriação, onde $91,5 \%$ do total de indivíduos responderam positivamente à questão.

\subsection{Resultados dos construtos}

Com base na escala do tipo Likert de 5 pontos, variando entre 1 (discordo totalmente) e 5 (concordo totalmente), os resultados para cada construto investigado são detalhados. Com relação ao cenário experimental apresentado aos entrevistados, expondo uma situação hipotética para conduzir as respostas dos três construtos avaliados engajamento, antecipação da satisfação e intenção de compra, a maior média encontrada para os Grupos 1 e 2 , de brasileiros e de norte-americanos - refere-se ao envolvimento de João com a compra realizada $(4,60$ e 4,45). Já a menor média, para o G1 $(4,35)$, relaciona-se ao engajamento de João, enquanto que para o $\mathrm{G} 2(4,20)$, indica o quanto João se sentiu estimulado com a compra.

De forma geral, os respondentes dos três grupos do Brasil se mostraram mais engajados do que os três grupos pesquisados dos EUA, com médias de 4,49; 4,53; 4,48 e 4,32; 4,16; 4,38 respectivamente. A análise paramétrica do construto, através do teste $t$, mostrou que há diferença significante entre os Grupos $\mathrm{G} 1$ e $\mathrm{G} 2(\mathrm{t}=2,146 ; \mathrm{p}=0,032)$. Esse dado evidencia que o nível de engajamento de brasileiros é significantemente maior que o de norte-americanos. Contudo, não há diferença significante entre os subgrupos dessa pesquisa.

O segundo construto analisado após a apresentação do cenário experimental foi a antecipação da satisfação. Os dois grandes grupos obtiveram suas maiores e menores médias sobre as mesmas afirmações, no que diz respeito à felicidade e à sensatez de João, respectivamente. Para o G1, a maior média foi de 4,28 e a menor, 3,36. Para o G2, a maior média foi de 4,20 , enquanto que a menor média foi de 3,73 . Considerando a média geral de todas as afirmações pertencentes a esse construto, a média dos norte-americanos do $\mathrm{G} 2(4,01)$ é maior do que a média dos brasileiros do $\mathrm{G} 1(3,93)$.

O teste $t$ realizado identificou que não há diferença significante entre $\mathrm{G} 1$ e $\mathrm{G} 2(\mathrm{t}=-0,26 ; \mathrm{p}=0,306)$, ou seja, apesar de os norte-americanos demonstrarem uma antecipação da satisfação mais alta, a diferença entre os grupos não é estaticamente significante. Da mesma forma, entre os subgrupos $\mathrm{G} 1.1$ e G1.2, e G2.2 e G2.2, não há diferença significante.

Por fim, para mensuração da intenção de compra dos respondentes, os Grupos $\mathrm{G} 1$ e G2 obtiveram suas maiores médias $(4,07$ e 4,01) sobre a mesma afirmação: É provável que João visite o mesmo website (que vende apenas produtos customizados) para fazer compras. A menor média do G1 (3,70) encontra-se na afirmação: Se João comprar um tênis novamente, ele provavelmente comprará no mesmo website (que vende apenas produtos customizados). $\mathrm{E}$ a menor média para o G2 $(3,95)$ na afirmação: Se João desejar comprar um novo tênis no futuro, ele provavelmente irá considerar comprar no mesmo website (que vende apenas produtos customizados). Considerando a média geral, formada por todas as afirmações do construto, os respondentes dos EUA (G2) obtiveram média maior $(3,98)$ do que o Grupo $1(3,87)$, formado por brasileiros.

A análise do teste $t$ de amostras independentes para igualdade de médias indicou que não há diferença significante entre os Grupos $\mathrm{G} 1$ e $\mathrm{G} 2(\mathrm{t}=-1,352 ; \mathrm{p}=0,177)$ quanto ao construto intenção de compra, assim como também não há diferença entre os subgrupos de norte-americanos e de brasileiros.

\section{DISCUSSÃO DOS RESULTADOS}

A partir das análises descritivas e paramétricas realizadas, inicialmente, percebe-se que, em relação a idade dos consumidores e a existência da experiência de cocriação, nota-se que, no Brasil, a média do grupo dos respondentes que já experimentaram a cocriação ( 24,09 anos) é superior à média daqueles que nunca tiveram a experiência $(22,47$ anos), contrariando os resultados obtidos nos EUA, onde os indivíduos mais jovens $(31,83)$ já experienciaram a cocriação e os mais velhos (34,17 anos) não. No caso dos norte-americanos é importante levar em conta o quanto plataformas on-line são favoráveis para a promoção de experiências de cocriação (BLASCO-ARCAS; HERNANDEZORTEGA; JIMENEZ-MARTINEZ, 2014) aliadas ao fato de que quanto mais jovem for o consumidor, mais integrado às ferramentas tecnológicas de consumo ele poderá ser considerado (THOMSON; LAING, 2003).

Essa perspectiva também é válida para os respondentes brasileiros, já que a média geral de idade destes (22,84 anos) é inferior à média de idade geral do grupo dos EUA (33,53 anos). Contudo, vale ressaltar a identificação de disparidade no poder de compra dos consumidores das duas sociedades, que é o foco da pesquisa, considerando questões socioeconômicas distintas do Brasil e também dos EUA, com base em informações levantadas pelo The World Factbook (CIA, 2018). Ainda a respeito do perfil de compra dos respondentes, pode-se notar que, tanto consumidores brasileiros quanto consumidores norte-americanos que já tiveram experiência prévia de cocriação, estão dispostos a pagarem mais caro ( $\mathrm{R} \$ 272,00$ e $\$ 86,00$, respectivamente) por um par de tênis (leia-se produto) novo do que aqueles que afirmaram não terem experimentado a cocriação previamente.

Retomando a teoria de Vargo e Lusch (2008) dizem que o valor percebido de uma marca ou de um produto é determinado a partir das próprias interações e experiências do consumidor e associadas a um comportamento do mesmo orientado para o relacionamento criado através da cocriação; enquanto Prahalad e Ramaswamy (2004) complementam 
afirmando que a proatividade do consumidor é a chave para criação e, sobretudo, para a percepção de valor.

Em relação ao (re)conhecimento do conceito de cocriação, os resultados apresentados pelos respondentes do Brasil e dos EUA são inversamente proporcionais, uma vez que a maioria dos brasileiros $(63,3 \%)$ respondeu de forma negativa à pergunta, e, por outro lado, $64 \%$ dos respondentes norte-americanos afirmaram conhecê-lo. Sendo assim, pode-se afirmar que o cenário apresentado na pesquisa foi suficientemente claro para os norte-americanos e possivelmente careceu de contextualização para os brasileiros, já esclarecido por Hall (1989), que caracterizou as sociedades através da comunicação verbalizada e da decodificação de mensagens.

Existem diferenças e similaridades entre brasileiros e norte-americanos no âmbito comportamental em um cenário de cocriação, pois as respostas comportamentais do consumidor, além de variarem pelos estímulos gerados através de experiências de cocriação, também dependem dos hábitos de consumo de produto/serviço, da orientação cultural e dos valores éticos de cada indivíduo (SOARES; FARHANGMEHR; SHOHAMB, 2007).

Com relação aos construtos investigados, a partir das médias encontradas nos dois grupos e a análise de significância realizada para cada um dos aspectos pesquisados (engajamento, antecipação da satisfação e intenção de compra), verificou-se que apenas um dos três comportamentos de consumo apresenta diferença significante comparando respondentes brasileiros e norte-americanos (Quadro 1).

Quadro 1 - Resultados dos construtos analisados

\begin{tabular}{|l|c|c|c|l|}
\hline \multirow{2}{*}{ CONSTRUTO } & \multicolumn{2}{|c|}{ MÉDIAS } & \multirow{2}{*}{ SIGNIFICÂNCIA } & \multirow{2}{*}{ DESCRIÇÃO } \\
\cline { 2 - 3 } & BRA & EUA & Ba & \\
\cline { 2 - 3 } & G1 & G2 & & $\begin{array}{l}\text { Brasileiros apresentam média significantemente maior em relação } \\
\text { aos norte-americanos }\end{array}$ \\
\hline Engajamento & 4,49 & 4,32 & 0,032 & Não existe diferença significante entre brasileiros e norte-americanos \\
\hline Antecipação da satisfação & 3,93 & 4,01 & 0,306 & Não existe diferença significante entre brasileiros e norte-americanos \\
\hline Intenção de compra & 3,87 & 3,98 & 0,177 &
\end{tabular}

Fonte: elaborado pelos autores (2019)

De acordo com os resultados, ambos os grupos obtiveram médias altas quanto ao engajamento do consumidor com a compra realizada através da experiência de cocriação, porém, os brasileiros apresentaram uma média significantemente maior quando comparados aos norte-americanos. Visto que a cocriação ocorre quando o cliente participa espontaneamente do relacionamento de troca consumidor-empresa-consumidor, o resultado da pesquisa permite afirmar que os respondentes brasileiros apresentaram comportamento de engajamento alinhado aos aspectos da cocriação como, por exemplo, a personalização única de uma experiência ou o compartilhamento de informações que auxiliem outros clientes a consumirem melhor (VAN DOORN et al., 2010). Aliado a isso, vale destacar que as médias obtidas para esse construto $(\mathrm{G} 1=4,49$ e $\mathrm{G} 2=4,32)$ são as mais altas dentre os três comportamentos de consumo pesquisados, o que consolida a relação intrínseca e direta entre as ações praticadas pelos indivíduos inseridos em um contexto de consumo e os comportamentos derivados de uma experiência de cocriação real (VAN DOORN et al., 2010).

Ainda, apesar dos brasileiros se mostrarem significantemente mais engajados com a experiência de cocriação proposta, os consumidores norte-americanos apresentaram médias mais altas ligadas à antecipação da satisfação e à intenção de compra. As diferenças entre o contexto socioeconômico e cultural dos EUA e do Brasil podem constituir possíveis explicações para o resultado encontrado, tendo em vista as diferenças econômicas de cada país. Ademais, como já mencionado, constatou-se que consumidores norte-americanos estão mais conscientes do que o conceito de cocriação engloba, sendo capazes de identificarem com facilidade quando são inseridos em uma experiência imersiva e colaborativa de cocriação.

Para Sreen, Purbey e Sadarangani (2018), os principais motivos que afetam negativamente a intenção e a decisão de compra dos consumidores são: o nível de preço do produto em questão (preços considerados altos/caros), a disponibilidade do mesmo e também a falta de conhecimento sobre o produto. Corroborando, Verleye (2015) pontua a relação positiva entre os níveis de conectividade e compartilhamento de informações e experiências e os níveis de antecipação da satisfação dos clientes com os produtos, consequentemente influenciando na intenção de compra do mesmo (LÓPEZ; MAYA; LÓPEZ, 2017).

Embora a revelação de que os dois grupos são praticamente equivalentes em suas respostas de consumo, uma vez que não há diferença significante entre os construtos analisados, a não ser pelo comportamento de engajamento, tanto brasileiros quanto norte-americanos apresentaram médias superiores a 3,0 (ponto neutro da escala) quando expostos às afirmações que mediram engajamento, antecipação da satisfação e intenção de compra. Logo, pode-se dizer que no contexto estudado (cocriação de um tênis), o valor percebido criado pela experiência on-line de cocriação é capaz de influenciar positivamente o comportamento de compra de indivíduos do Brasil e dos EUA (PRAHALAD; RAMASWAMY, 2004; LÓPEZ; MAYA; LÓPEZ, 2017). 


\section{CONSIDERAÇÕES FINAIS}

O propósito desta pesquisa foi analisar respostas comportamentais de consumo em um cenário experimental de cocriação em dois grupos culturais distintos. De forma geral, constatou-se que tanto brasileiros quanto norteamericanos têm uma percepção positiva a respeito da cocriação. Adicionalmente, os brasileiros são significantemente mais engajados do que os norte-americanos. Contudo, não foram identificadas diferenças significativas entre os respondentes de cada país quanto à antecipação da satisfação causada pela experiência de cocriação e à intenção de compra de um produto customizado.

Existem fatores que podem ter influenciado a pesquisa, bem como podem ter sido responsáveis sobre como cada público reage a experiências de cocriação de valor como: conhecimento e familiarização que se têm com a prática, o perfil de consumo dos indivíduos e até mesmo o perfil demográfico, que abrange aspectos de gênero, faixa etária, grau de escolaridade e status econômico. Somado a isso está a falta de experiência real da situação exposta no texto, que pode ter influenciado na avaliação dos elementos abordados.

Frente a esse cenário, as análises apresentadas constituem uma ferramenta que auxilia no processo de entendimento das características culturais de consumidores de países diferentes em um cenário de cocriação. Empresas, tanto nacionais quanto internacionais, bem como profissionais do comércio exterior que possuem relações comerciais com os países participantes da pesquisa, ou de mesma orientação cultural, podem utilizar os dados levantados para auxiliar no gerenciamento de suas estratégias de inovação e comunicação, de forma a implementarem ferramentas inovadoras de cocriação bem-sucedidas.

Como implicações gerenciais desta pesquisa, os resultados obtidos baseados em consumidores brasileiros e dos EUA, no contexto de customização de um tênis, constitui uma ferramenta de análise para as estratégias de marketing e inovação de empresas voltadas para a produção de bens, sobretudo, calçadistas, que atuam nos mercados do Brasil e nos EUA. Dessa forma, é possível que os profissionais de marketing considerem as análises realizadas visando melhores resultados, comparando-as com as estratégias atuais e adaptando-as.

Com relação ao engajamento, as empresas presentes nos EUA deveriam dedicar mais esforços para engajar os consumidores do que as empresas presentes no Brasil. Assim, sugere-se que essas empresas realizem pesquisas que identifiquem quais estímulos adicionais têm maior aceitação dos norte-americanos para que a experiência seja adaptada e atinja níveis de engajamento mais altos.

Apesar de as médias de intenção de compra terem sido positivas nos dois grupos culturais, os brasileiros se mostraram menos propensos para tal em comparação aos norte-americanos. Levando em consideração que os consumidores do Brasil são mais engajados que os dos EUA, as empresas brasileiras podem promover ações de incentivo ao consumo, como a criação de campanhas promocionais cumulativas que estimulem os clientes a efetivarem a compra, como, por exemplo, pague 2 leve 3 ou o combo desconto nos produtos e frete grátis no caso de lojas virtual.

Como limitações desta pesquisa, identificou-se a escolha de um contexto específico para o cenário experimental - customização de um tênis - que pode ter influenciado as respostas dos participantes, baseando-as em experiências e conceitos prévios dos mesmos em relação à essa categoria de produto. Nesse sentido, é interessante que pesquisas futuras avaliem outros formatos de cocriação. Por fim, e ainda com relação aos participantes da pesquisa, sabendo que as amostras obtidas entre as nacionalidades apresentaram médias de idade distantes entre si, sugere-se realizar uma comparação entre faixas etárias em relação à percepção sobre atividades de cocriação.

\section{REFERÊNCIAS}

ARNOULD, E. J.; THOMPSON, C. J. Consumer Culture Theory (CCT): Twenty Years of Research. Journal of Consumer Research, [s. I.], v. 31, n. 4, p. 868-882, 2005. Link. Acesso em: 7 jul. 2018.

BELCH, G.; BELCH, M. Advertising and Promotion: an Integrated Marketing Communications Perspective. New York: The McGraw-Hill Companies, 2004.

BLASCO-ARCAS, L.; HERNANDEZ-ORTEGA, B.; JIMENEZ-MARTINEZ J. The online purchase as a context for co-creatingexperiences: Drivers ofandconsequences for customerbehavior. Internet Research, [s. I.], v. 24, n. 3, p. 393-412, 2014. Link. Acesso em: 3 jul. 2018

BONE, P. F.; ELLEN, P. S. The Generation and Consequences of Communication-Evokedlmagery. Journal of Consumer Research, [s. I.], v. 19, n. 1, p. 93-104, 1992. Link. Acesso em: 14 ago. 2018.

BRODIE, R. J. B. et al.Customer Engagement: Conceptual Domain, Fundamental Propositions, and Implications for Research. Journal of Service Research, [s. I.], v. 14, n. 3, p. 252-271, 2011. Link. Acesso em: 9 jul. 2018.

BUSSER, J. A.; SHULGA, L. V. Co-created value: Multidimensional scale and nomological network. Tourism 
Management, [s. I.], v. 65, n. 1, p. 69-86, 2018. Link. Acesso em: 12 out. 2018.

CENTRAL INTELLIGENCE AGENCY. The World Factbook. [S. I.]: CIA, 2018. Link. Acesso em: 22 abr. 2018.

CIAN, L.; KRISHNA, A.; ELDER, R. S. This Logo Moves Me: Dynamic Imagery from Static Images. Journal of Marketing Research, [s. I.], v. 51, n. 2, p. 184-197, 2014. Link. Acesso em: 14 ago. 2018.

COSSíO-SILVA, F. et al. Value co-creation and customer loyalty. Journal of Business Research, [s. I.], v. 69, n. 5, p. 1621-1625, 2016. Link. Acesso em: 10 jul. 2018.

FINSTERWALDER, J. A 360-degree view of actor engagement in service co-creation. Journal of Retailing and Consumer Services, [s. I.], v. 40, n. 1, p. 276-278, 2018. Link. Acesso em: 9 jul. 2018.

FRANCE, C. et al.Customer brand co-creation behavior: conceptualization and empirical validation. Marketing Intelligence \& Planning, [s. I.], v. 36, n. 3, p. 334-348, 2018. Link. Acesso em: 9 jul. 2018.

FÜLLER, J. Refining Virtual Co-Creation from a Consumer Perspective. California Management Review, [s. I.], v. 52, n. 2, p. 98-122, 2010. Link. Acesso em: 10 abr. 2018.

HAIR, J. F. et al. Fundamentos de métodos de pesquisa em administração. Porto Alegre: Bookman, 2005.

HALL, E. T. Beyondculture. New York: Anchor Books, 1989.

HELKKULA, A. Characterising the concept of service experience. Journal of Service Management, [s. I.], v. 22, n. 3, p. 367-389, 2011. Link. Acesso em: 9 jul. 2018.

HOFSTEDE, G.; HOFSTEDE, G. J.; MINKOV, M. CulturesandOrganizations:Softwareofthe mind: Intercultural cooperationand its importance for survival. 3. ed. New York, NY: McGraw-Hill, 2010.

IND, N.; COATES, N. The meanings of co-creation. European Business Review, [s. I.], v. 25, n. 1, p. 86-95, 2013. Link. Acesso em: 12 out. 2018.

JAAKKOLA, E.; HELKKULA, A.; AARIKKA-STENROOS, L. Service experience co-creation: conceptualization, implications, and future research directions. Journalof Service Management, [s. I.], v. 26, n. 2, p. 182-205, 2015. Link. Acesso em: 7 jul. 2018.

JEONG, J. Y.; CROMPTON, J. L. Do subjects from high and low context cultures attribute different meanings to tourism services with 9-ending prices? Tourism Management, [s. I.], v. 64, n. 1, p. 110-118, 2018. Link. Acesso em: 17 jul. 2018.

JIMÉNEZ-BARRETO, J.; CAMPO-MARTÍNEZ, S. Destination website quality, users' attitudes and the willingness to participate in online co-creation experiences. European Journal of Management and Business Economics, [s. I.], v. 27, n. 1, p. 26-41, 2018. Link. Acesso em: 5 jul. 2018.

KIM, D.; PAN, Y.; PARK, H. S. High- Versus Low-Context Culture: A Comparison of Chinese, Korean, and American Cultures. Psychology \& Marketing, [s. I.], v. 15, n. 6, p. 507-521, 1998. Link. Acesso em: 04 maio 2018.

KLEIN, A. Z. et al. Metodologia de pesquisa em administração: uma abordagem prática. São Paulo: Atlas, 2015.

LÓPEZ, R. A.; MAYA, S. R. de; LÓPEZ, I. L. Sharing co-creation experiences contributes to consumer satisfaction. Online Information Review, [s. I.], v. 41, n. 7, p. 969-984, 2017. Link. Acesso em: 7 jul. 2018.

LU, B.; FAN, W.; ZHOU, M. Social presence, trust, and social commerce purchase intention. Computers in Human Behavior, [s. I.], v. 56, n. 1, p. 225-237, 2016. Link. Acesso em: 25 jul. 2018.

LUO, N.; ZHANG, M.; LIU, W. The effects of value co-creation practices on building harmonious brand community and achieving brand loyalty on social media in China. Computers in Human Behavior, [s. I.], v. 48, n. 1, p. 492-499, 2015. Link. Acesso em: 25 jul. 2018.

MATHIS, E. F. et al. The effect of co-creation experience on outcome variable. Annals of Tourism Research, [s. I.], 
v. 57, n. 1, p. 62-75, 2016. Link. Acesso em: 5 jul. 2018.

NGUYEN, A.; HEELER, R. M.; TARAN, Z. High-low context cultures and price-ending practices. Journal of Product \& Brand Management, [s. I.], v. 16, n. 3, p. 206-214, 2007. Link. Acesso em: 3 jul. 2018.

PAYNE, A. F.; STORBACKAB, K.; FROW, P. Managing the co-creation of value. Journal of the Academy of Marketing Science, [s. I.], v. 36, n. 1, p. 83-96, 2008. Link. Acesso em: 2 jul. 2018.

PAYNE, A. F. et al. Co-creating brands: Diagnosing and designing the relationship experience. Journal of Business Research, [s. I.], v. 62, n. 3, p. 379-389, 2009.

PHILIPPE, A.; GUINTCHEVA, G. Using Affect-Expectations Theory to Explain the Direction of the Impacts of Experiential Emotions on Satisfaction. Psychology \& Marketing, [s. I.], v. 31, n. 10, p. 900-913, 2014. Link. Acesso em: 5 jul. 2018.

PRAHALAD, C. K.; RAMASWAMY, V. O futuro da competição:como desenvolver diferenciais inovadores em parceria com os clientes. Rio de Janeiro: Elsevier, 2004.

RAMASWAMY, V.; OZCANB, K. What is co-creation? An interactional creation framework and its implications for value creation. Journal of Business Research, [s. I.], v. 84, n. 1, p. 196-205, 2018. Link. Acesso em: 4 mai. 2018.

SAMARA, B. S.; BARROS, J. C. Pesquisa de marketing: conceitos e metodologia. 4. ed. São Paulo: Pearson, 2007.

SHIV, B.; HUBER, J. The Impact of Anticipating Satisfaction on Consumer Choice. Journal of Consumer Research, [s. I.], v. 27, n. 2, p. 202-216, 2000. Link. Acesso em: 14 ago. 2018.

SOARES, A. M.; FARHANGMEHR, M.; SHOHAMB, A. Hofstede's dimensions of culture in international marketing studies. Journal of Business Research, [s. I.], v. 60, n. 3, p. 277-284, 2007.

SREEN, N.; PURBEY, S.; SADARANGANI, P. Impact of culture, behavior and gender on green purchase intention. Journal of Retailing and Consumer Services, [s. I.], v. 41, n. 1, p. 177-189, 2018.

THOMSON, E. S.; LAING, A. W. "The Net Generation": Children and Young People, the Internet and Online Shopping. Journal of Marketing Management, [s. I.], v. 19, n. 3-4, p. 491-512, 2003.

VAN DOORN, J. et al. Customer engagement behavior: Theoretical foundations and research directions. Journal of Service Research, [s. I.], v. 13, n. 3, p. 253-266, 2010.

VARGO, S. L.; LUSCH, R. F. Service-dominant logic: continuing the evolution. Journal of the Academy of Marketing Science, [s. I.], v. 36, n. 1, p. 1-10, 2008.

VERLEYE, K. The co-creation experience from the customer perspective: its measurement and determinants. Journal of Service Management, [s. I.], v. 26, n. 2, p. 321-342, 2015.

YI, Y.; GONG, T. Customer value co-creation behavior: Scale development and validation. Journal of Business Research, [s. I.], v. 66, n. 9, p. 1279- 1284, 2013. 


\section{Contato:}

Nicole Speguem Muratore

E-mail: nmuratore@ucs.br

\section{Fernanda Lazzari}

E-mail: fernandalazzari@hotmail.com

Juliana Matte

E-mail: ju.cxs1@gmail.com

Vandoir Welchen

E-mail: vwelchen@ucs.br 